\title{
PROCEEDINGS
}

\author{
DECUS Conference-Fifth European Seminar
}

The papers to follow were presented at the Fifth European Seminar of the Digital Equipment Computer Users Society (DECUS) held at Stockholm, Sweden. The reports are printed here through the cooperation of Mrs. A. J. Cossette, Executive Director of the Society.

\section{A simple time-sharing system for speech perception experiments}

\section{W. A. AINSWORTH and J, B. MILLAR* \\ Department of Communication, University of Keele, Staffordshire, England}

Much of our knowledge of speech perception has been obtained by psychophysical methods using speech synthesizers (Cooper et al, 1952). In those experiments a number of sounds of known composition are presented to a group of listeners who are required to try to recognize the sounds as spoken words or to judge their quality. By analysis of the listeners' responses, it is possible to infer the perceptual importance of the various acoustic features of the stimulus sounds.

Human speech is an extremely complex subject, and the number of experiments of this kind which might be performed is vast. The form of the experiments, however, is fairly standard, so the use of a computer is appropriate. The proportion of the computer's time spent in servicing an experiment of this type is rather low, so it is economical to introduce time sharing in order that other programs may be run concurrently with the experimental program. A system has been developed which enables a background program to be run in one field of an $8 \mathrm{~K}$ PDP-8 while the foreground program in the other field controls the experiment.

\section{MODULAR PROGR AMMING}

The configuration used for these tasks consists of a PDP 8 with $8 \mathrm{~K}$ words of core, high-speed paper-tape reader and punch, Teletype, two Dectape units, a 338 display, an A-D converter, and D-A outputs. The computer is used for analyzing and synthesizing speech, analysis of physiological data, generation of visual and a coustic stimuli for psychophysical experiments, and for statistical analysis of data.

*We are indebted to the many members of the Department of Communication, both past and present, whose ideas and contributions have helped in the evolution of the present system. The work was supported by the Science Research Council.
In a research laboratory where all these activities are in progress and where the experiments are continually changing, the computer rapidly becomes overloaded. One reason for this is the amount of time spent in developing programs. This problem has been overcome to some extent by writing 30-40 subroutines or program modules which perform all the common tasks, such as handling peripherals and sorting data. These modules have been written in such a way that they can readily be combined to produce useful programs with the minimum of debugging. Using the time-sharing system, they can be edited and assembled as a background job while the priority program is controlling an experiment.

The modules have been written in PAI 3 language. They are called from the main program by a sequence consisting of a JMS instruction followed by a list of parameter words which specify the exact task the module is to perform. For example, one module controls input and output via the reader, punch, and Teletype. One bit in the first word following the subroutine call specifies the direction of transfer of data, another bit high or low speed, another the single character or string of characters, etc. The second word specifies the location of the start of the character string. After the module has been executed, control returns to the location following the last parameter word of the subroutine call.

In order to enable the modules to be combined with the minimum of editing, all the labels and tags in a single module have the initial two letters in common. Modules have been written so that they are relocatable in any page in either field, and so that they can address data anywhere in the core. To facilitate packing and minimize indirect addressing, they have been written, wherever possible, to be an integral number of half pages in length.

\section{TIME-SHARING SYSTEM}

The time-sharing system which has been developed enables two programs to be run simultaneously on the computer. Each program occupies a single field of core. The foreground program which controls the experiment resides in Field 1 , and the background program in Field $\emptyset$. The peripherals are divided between the programs by agreement. In practice, this has not proved a great restriction as ADCs and DACs go naturally with foreground programs, whereas the background programs use paper-tape readers and punches. Each program normally has one Dectape unit available; the foreground program uses its unit for data storage, and the other unit is used for a library of alternative background programs which can be changed without interrupting the foreground program.

Time is shared between the two programs according to the following scheme. The foreground program is started and begins to run the experiment. When it reaches a point where it would normally have to wait (e.g., while it flashes a light or waits for an expeximental $S$ to press a switch), control passes to the background program for $1 / 2 \mathrm{msec}$ and then returns to the foreground program. This time is short enough for the experimental $S$ to think that the computer responds immediately to his actions. Provided that the foreground program is such that it would normally be "waiting" for a large proportion of its time, the background program runs at a reasonable speed. A practical criterion is that a person using an editor as a background program should not be aware that another program is being executed simultaneously.

In order to implement this system, two additional pieces of hardware have been added to the computer: an interrupt handler and a clock. The clock is merely a countdown register which causes a program interrupt 500 microsec after it has been started. The interrupt handler was originally added to the computer for another purpose, but it has the useful property 


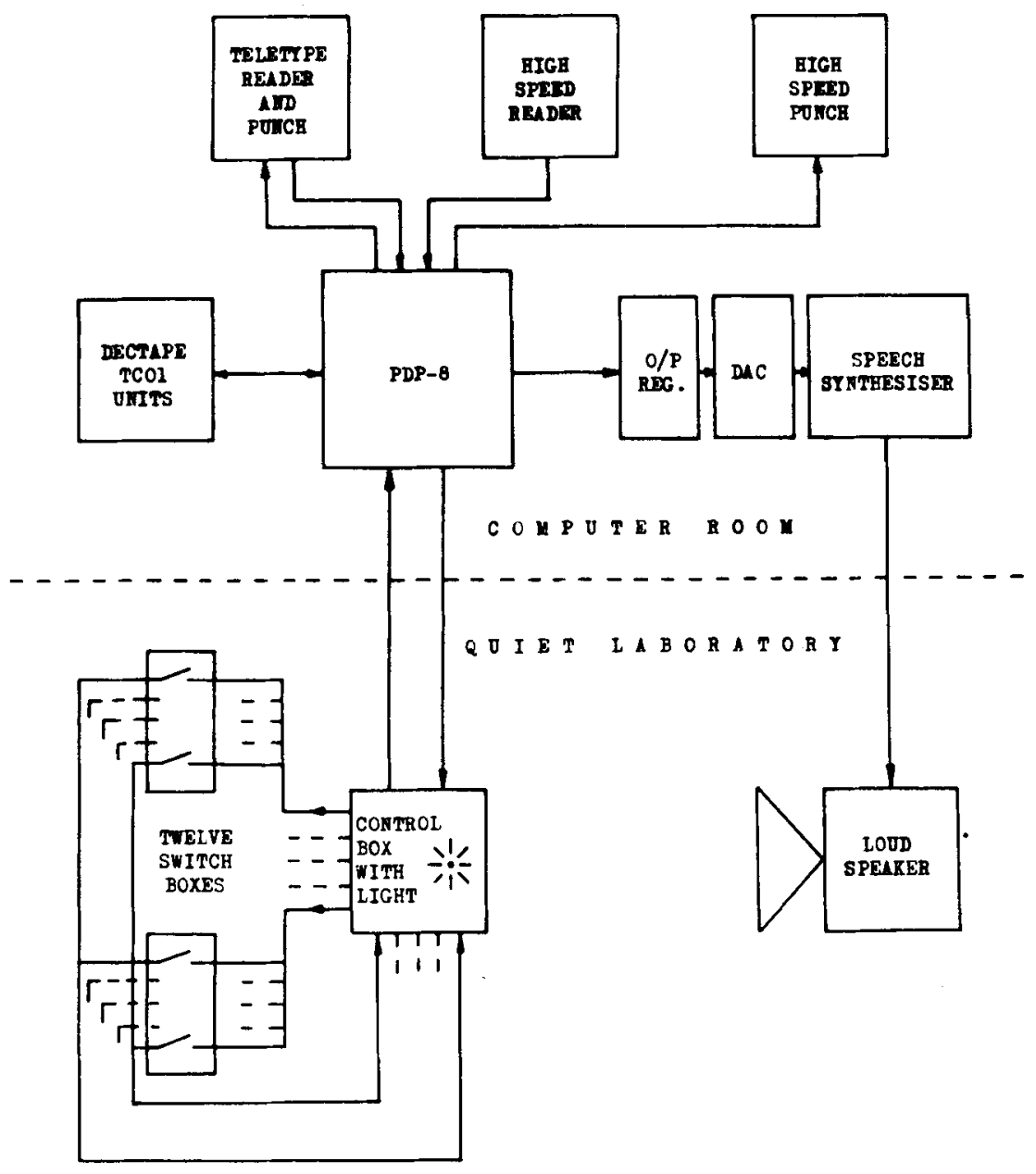

Fig. 1. Apparatus used in the experiment.

of disenabling flags from the interrupt bus by program control. The only software which was written for the time-sharing system is a half-page module, called IR, which handles the jumping between fields, disenables certain flags from the interrupt bus, and controls the clock.

Foreground programs are written in PAL 3 , according to the normal rules but with the following restrictions: (1) The program must contain the control module IR in Locations 7500-7577. (2) The program must not contain an HLT instruction. (3) It must not use the clock. (4) It may use interrupts provided the corresponding flags are not used in noninterrupt mode by the background program. This normally excludes the Teletype, high-speed reader and punch, and Dectape flags. The starting address of the interrupt handling routine must be put in Location 7577. (5) A foreground program must contain an effective JMS IRN instruction near its start. This initializes the time-sharing system. (6) External conditions are tested by means of the following sequence:
JMS IRST / GO TO FIELD $\emptyset$ for 500 uSEC

SKIP / SKIP IF CONDITION IS SATISFIED

JMP . -2 !

\section{JUMP BACK AND REPEAT}

(7) "Wait" loops should, if possible, be made multiples of 500 microsec in duration and should incorporate JMS IRST instructions to control timing. (8) Any part of a foreground program which runs for an appreciable time (greater than $500 \mathrm{msec}$ ) without JMS IRST instruction should, if possible, have one inserted. This will slow the program slightly, but will enable the foreground program to make some progress. (9) A foreground unit available (not Unit 8). When it uses a tape it should check that the other tape is not being used by the background program.

Background programs may also be written in PAL, except that the following restrictions apply: (1) They program normally has one Dectape must not use the clock. (2) They must not contain HLT instructions. (3) They must not use Locations $0-3$ inclusive. These locations must contain initially:

๑) $76 \emptyset \emptyset /$ STARTING ADDRESS OF LIBRARY SYSTEM

\section{1) 6213 / CHANGE TO FIELD 1}

2) $54 \emptyset 3 /$ JMP I 3

\section{3) $7524 /$ IROUT}

(4) The program in Field $\emptyset$ can be changed by means of a modified library system. Exit to the library system is accomplished by:

\section{JMP I LIB \\ LIB, $76 \emptyset \emptyset$}

(5) A background program is called by typing its name. Failures occur if the foreground program is taking up too great a proportion of the time. If a failure does occur, control will be returned to the library system and the call should be repeated.

Among the system programs which have been modified to run as background programs are the $4 \mathrm{~K}$ Fortran compiler and operating system. It is therefore possible to write background programs in Fortran.

\section{SPEECH PERCEPTION EXPERIMENTS}

In order to illustrate the use of the system, a typical experiment will be described. A number of speech-like sounds are to be synthesized and presented in random order to a group of listeners. The listeners are asked to try to recognize the sounds and to press the most appropriately labeled switch on the box in front of them. The computer records the responses and stores them in systematic order for later analysis.

A diagram of the apparatus is shown in Fig. 1. The speech synthesizer is of the parallel formant type (Holmes, Mattingly, \& Shearme, 1967), and is controlled by analogue voltages derived from a set of DACs connected to the accumulator of the PDP-8 via a 6 -bit input/output register. The loudspeaker is housed in the ceiling of a quiet laboratory some distance from the computer room. Up to 12 listeners can take part in a session. Each listener is provided with a box containing 12 switches which are labeled appropriately for the current experiment (Ainsworth, 1968). The experimenter uses one of these boxes (SBI) to communicate with the computer. 
At the beginning of the experiment a tape recording is played to instruct the listeners. The experimenter presses a switch and the session begins. The listeners hear a low-pitched hiss followed by a speech-like sound. They set their switches and, when one switch on each box has been set, they hear a high-pitched hiss. This is the signal to reset the switches. This sequence is repeated until the predetermined set of sounds has been exhausted. The experimenter can then signal for a new experiment to be started.

A flow diagram of the program which generates the control signals for the speech synthesizer and records the responses of the listeners is shown in Fig. 2. The attachment of a small box labeled IR to a larger box means that while the current task is being carried out control passes to the background program for some part of the time.

First of all, the IR module is initialized as explained in Section 3. Once this has been done, the person in the computer room can begin to use the background programs. When the experimenter signals that the session is to start, the random-number generator, which determines the order in which the sounds are to be presented, is initialized. Then the computer reads the experimenter's switches (SBI) again to determine which of the switch boxes are to be used in the experiment. When it has done this, it checks to see that all the switches on these boxes are cleared, and if they are not, it flashes a light.

Reading switches is accomplished by setting a bit in the accumulator (to determine the box to be read) then giving an IO instruction which sends a pulse to that particular switch box. The pulse is made 500 microsec in duration by executing a JMS IRST instruction. After this time has elapsed, the bits in the accumulator corresponding to the closed switches on this box will be set. Light flashing is accomplished by setting a bit in an IO register, then clearing it $100 \mathrm{msec}$ later, say, after jumping to the background program 20 times.

Having initialized all the counters and checked that the switches are cleared, the next task is to assemble the control signals for the synthesizer. It is sometimes desirable to intermingle standard and experimental sounds in a single session in order to try to keep a check on the listeners' perceptual reference frames during the course of an experiment (Ainsworth \& Millar, 1971). The random-number. generator is used to decide which particular stimulus is to be presented next.

The warning hiss is given followed by the speech sound. The control

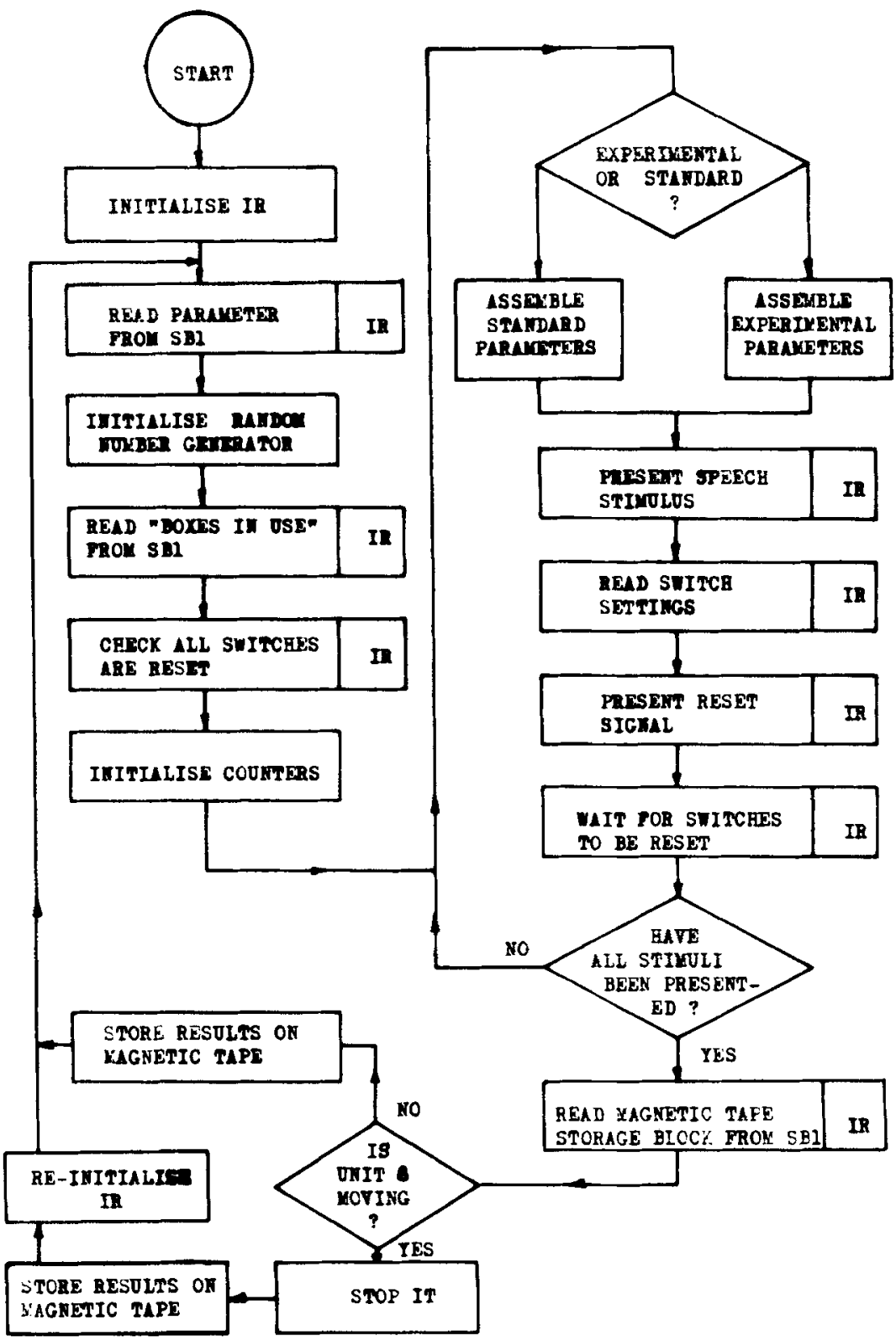

Fig. 2. Flow diagram of program used for controlling the experiment.

signals for the synthesizer are derived from pulses from an IO register via the DACs. The pulses, again, are 500 microsec in length, thus enabling the background program user to have a share of the computer time even while the sounds are being generated.

The computer waits for all the listeners to respond, transferring control in and out of the background program several times while it is waiting. It then reads the responses and stores them, gives the signal for the switches to be reset, and goes to the background program while this is done. Finally, it checks to see if all the stimuli have been presented and, if not, it returns to the random-number generator to determine which to present next.

When all the stimuli have been presented, the computer informs the experimenter by flashing a light. The experimenter uses his switches to indicate where on tape he wants the responses stored. The computer stores the responses on Dectape, checking that the other tape unit was not in motion. If it was, it stops it, then reinitializes IR after it has finished. The computer is then ready for another experimental session, with the sounds presented in a different order or with some other parameter changed.

\section{BACKGROUND PROGRAMS}

\section{PAL 3 Assembler}

The PAL 3 assembler has been modified to run as a background program. Pass 1 is executed by calling the program. The symbol table is 
punched out by the high-speed punch and may be listed off-line if desired. The second and third passes are executed by typing $\mathrm{P} 2$ and $\mathrm{P} 3$, respectively. Output in each case is via the high-speed punch. Between passes, control returns to the library system so assembly may be terminated after any pass.

\section{Symbolic Editor}

The editor may also be run as a background program. Once it has been called it waits with an empty buffer until it receives instructions from the Teletype. The command structure is unchanged except for the addition of a new command, E, which causes exit from the editor to the library system.

\section{Fortran}

The Fortran system has been modified so that high- or low-speed input and output is determined by the form of the statements instead of the settings of the switch register.

The compiler operates as a background program using only high-speed input and output. Error diagnostics must be read off-line from the end of the output tape.
Symbolprint is executed if it is called immediately after compilation.

Calling the operating system merely loads the program into the machine. "GO" must be typed in order to start the program. The program may be repeated any number of times by this command. "PAUSE" statements return control to the library system and "CONT" must be typed in order to continue execution. All Fortran programs must contain a "STOP" statement so that control will eventually return to the library system. If the operating system detects an error at run-time, the diagnostic is printed by the Teletype and control returns to the library system. No recovery is possible.

\section{CONCLUSION}

Two ways of increasing the effectiveness of a small computer in a multiuser research laboraory have been described. Programming has been simplified by the use of modules, and the effective time available on the machine has been increased by the introduction of a simple form of time sharing. The system has been described in terms of a program for controlling a speech perception experiment, but this merely reflects the interests of the authors. The techniques can be applied equally readily to other psychophysical experiments, to the processing of physiological data on-line, and probably to many other situations where the machinery to be controlled operates at human rather than computer speeds.

\section{REFERENCES}

AINSWORTH, W. A. A speech synthesizer controlled by a $P D P-8 / 388$. Proceedings of the Third Decus European Seminar, $1967,1-4$.

AINSWORTH, W. A. First formant transitions and the perception of synthetic semivowels. Journal of the Acoustical Society of America, 1968, 44, 689-694.

AINSWORTH, W. A., \& MILLAR, J. B. Methodology of experiments on the perception of synthesized vowels. Language \& Speech, December 1971, in press.

COOPER, S., DELATTRE, P. C. LIBERMAN, A. M., BORST, J. M., \& GERSTMAN, L. J. Some experiments on the perception of synthetic speech sounds. Journal of the Acoustical Society of America, 1952, 24, 597-606.

HOLMES, J. N., MATTINGLY, I. G., \& SHEARME, J. N. Speech synthesis by rule. Language \& Speech, 1967, 3, 127-143.

\section{LINC-8 presents and controls visual experiments*}

\section{GEOFF CUMMING \\ Institute of Experimental Psychology, Oxford, England}

\section{INTRODUCTION \\ The Tachistoscope}

The desire to eliminate the effects of eye movements and to control the presentation time of material used in early span-of-apprehension and visual perception experiments led to the development of the tachistoscope (Woodworth \& Schlosberg, 1954). The most common modern tachistoscopes use electronic timers to switch fluorescent lamps which light one of two or three display fields. Partly silvered mirrors are arranged so that any lighted field appears straight ahead to the S. Material for presentation, such as letters or dot patterns or colored figures, is made up on white cards for mounting in the fields of the tachistoscope. This type of tachistoscope gives excellent definition and contrast of display material, and

* This paper is a later version of a paper presented at the 5th European Seminar of DECUS in Stockholm, September 1969, and published in the Proceedings. allows accuracy and flexibility in the choice of timing parameters. But the use of partly silvered mirrors severely limits the number of fields that may be used, because a sizable proportion of the light reflected from a field is lost every time it is transmitted or reflected by such a mirror, and the use of more fields entails the introduction of more such mirrors into the light paths from the fields to the S's eye. In practice, this means that only two or three arrays may be displayed in a single trial, and frequently one of these arrays must be a masking field, or "visual noise field," to reduce the persistence of afterimages of the preceding array. Also, it is difficult to align different arrays presented on a single trial exactly, as is required in many masking experiments. And it can be very time-consuming to make up the cards for presentation, and then tedious to shuffle them in and out of card holders and change timer settings between trials.

Various devices allowing more than three or four different arrays to be displayed in a single trial have been built. For example, Eriksen and Spencer (1969) used 19 timers, 10 lamps, and 10 optical wave guides to be able to present 10 letters or digits sequentially in one trial. Such devices lose the great flexibility in choice of type, color, size, and position of presentation of the material allowed by the use of cards in mirror tachistoscopes.

The development of television equipment introduced the possibility of displaying in sequence a large number of arrays on a single screen. For example, Averbach and Coriell (1961) used multichannel generating equipment to present three displays in quick succession on a television screen.

\section{Computer-Controlled CRT Displays}

Only with the application of the digital computer to the control of CRT displays was the "many-channel" tachistoscope made possible (Green, 1963). Two important and distinct types of flexibility are offered. First, computer control displays may be built up in space and time by the presentation of any number of elements or whole arrays, each with its own spacing and timing display parameters. Timing is controlled accurately, and different parts of the 